Écrire

l'histoire

\section{Écrire l'histoire}

Histoire, Littérature, Esthétique

18 | 2018

Révolution

\title{
Boussole. Entretien avec Nathalie Quintane
}

Par Marius Loris et Lise Wajeman

Nathalie Quintane, Marius Loris et Lise Wajeman

\section{CpenEdition}

Journals

Édition électronique

URL : http://journals.openedition.org/elh/1501

DOI : 10.4000/elh.1501

ISSN : 2492-7457

Éditeur

CNRS Éditions

\section{Édition imprimée}

Date de publication : 20 novembre 2018

Pagination : 187-194

ISBN : 978-2-271-12431-9

ISSN : 1967-7499

Référence électronique

Nathalie Quintane, Marius Loris et Lise Wajeman, « Boussole. Entretien avec Nathalie Quintane », Écrire l'histoire [En ligne], 18 | 2018, mis en ligne le 20 novembre 2018, consulté le 16 février 2021. URL : http://journals.openedition.org/elh/1501 ; DOI : https://doi.org/10.4000/elh.1501 


\title{
Boussole
}

\section{Entretien avec Marius Loris et Lise Wajeman}

\begin{abstract}
Avec Tomates (2010), Nathalie Quintane a planté d'incomparables fruits: un livre qui parvenait à réunir les militants de poésie contemporaine et les amateurs d'action politique. Ce n'est pourtant pas le premier livre de son auteure, évidemment pas le premier livre de son genre: mais il s'est manifesté là une intelligence du présent - le réel et la langue pour le dire - qui a fait date. Depuis, Nathalie Quintane est devenue

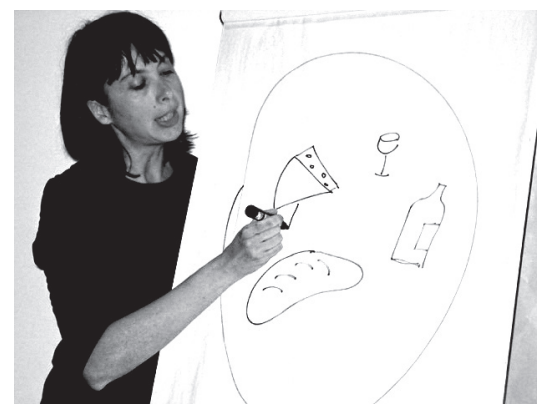

Nathalie Quintane un genre de boussole. Le contraire d'une figure de proue, à l'avant-garde: une boussole se laisse aimanter, et met toute son énergie à trouver, avec nous, comment s'orienter. "[Elle] a peut-être des secrets pour changer la vie ? Non, [elle] ne fait qu'en chercher, me répliquais-je » (Rimbaud, Une saison en enfer). Nathalie Quintane fait partie de cette génération de poètes qui ne sont pas certains d'être la révolution ${ }^{1}$, mais qui, tout de même, continuent à désirer aimanter l'action.
\end{abstract}

Ça signifie quoi pour vous, être un écrivain contemporain?

Ça ne veut pas dire la même chose que quand j'ai commencé. Ça me demande de faire un petit peu d'archéologie, mes débuts datent d'il y a vingt-cinq ans... Mes deux premiers livres sont sortis en même temps, en 1997, chez deux éditeurs différents : l'un, chez POL, s'appelle Chaussure et ne parle que de pied, de lacet, etc. Il est en fait issu de l'autre livre, Remarques
(Cheyne), qui, sous un format proche de celui de la plaquette, présentait une série de phrases courtes, presque tautologiques, sur des choses très quotidiennes... C'était post-pongien, disons, même si la source, en ce qui me concerne, venait plutôt de Lichtenberg et de Sei Shonagon. Dans ce livre figurait la remarque suivante: "Quand je marche, il y a toujours l'un de mes pieds qui a disparu derrière moi » : Chaussure est l'extension maximale de ça. 
C'était une sorte de commande implicite de POL : il n'avait pas pris Remarques, il voulait voir si j'en avais un peu plus sous la semelle. La quatrième de couverture de Chaussure est claire: "C'est un livre de poésie pas spécialement poétique, de celle (la poésie) qui ne se force pas. » Il s'agissait donc d'un livre de poésie littérale, qui entendait, comme la plupart des livres de mes camarades de l'époque, en terminer avec le renouveau lyrique qui avait pris place dans les années 1980 (Christian Bobin, etc.). Le «contemporain» prenait position là et, plus formellement ou techniquement, dans une reprise du cutup, du montage de textes, dans un souci plastique héritier des poésies visuelles et de l'attention portée à la mise en page, à la typographie, à l'aspect matériel et éditorial des choses. Le "contemporain" continue à creuser là, mais d'autres soucis sont venus s'agréger : des emprunts aux sciences sociales en matière de thème et de méthode, et un acharnement quant à l'usage de certains mots (les prépositions, par exemple).

Que s'est-il passé entre Chaussure (1997, donc) et Que faire des classes moyennes? (POL, 2016) ?

Il y a bien une continuité : je continue de penser qu'il suffit d'écrire un livre qui s'appelle Chaussure pour faire un acte politique. Sauf qu'il y a une dizaine d'années, j'ai eu le sentiment que ce n'était plus suffisant, qu'il fallait thématiser cette question. C'est presque une histoire de génération : ça faisait bientôt vingt ans que j'écrivais, et il y a ce moment fatal, quoi qu'on fasse d'ailleurs, où vingt ans ont passé, et l'époque a changé. Si tu ne saisis pas ton propre changement possible à l'intérieur de ce changement-là - tenant compte de ce changement -, ce n'est pas la peine. Perpétuer un logo, une marque, une écriture, c'est très déprimant.

Christian Prigent avait soulevé ce problème très tôt dans notre génération, en écrivant un livre qui portait Salut les anciens d'un côté et de l'autre, retourné, Salut les modernes. Le livre est sorti en 2000 : Salut les modernes était adressé à ma génération, et nommément à Christophe Tarkos, Philippe Beck, Charles Pennequin. La phrase qu'on a retenue de ce livre, c'était une question qui nous était adressée et qui demandait ce qu'on faisait avec nos revues, quelle en était la valeur d'usage - question clef qu'on ne se posait pas bien, selon Prigent, pas assez, de manière incomplète, trop brouillonne, ou prenant date avant tout pour telle écriture, telle singularité, telle "position»; à usage interne, en somme. C'était peut-être parce que nous en étions encore à nos débuts - on a tous commencé à publier vers 1994, avec une espèce $d$ 'inflation de publications, une forme de concurrence entre nous. Prigent avait raison de pointer un danger : ça risquait de se figer en marque de fabrique, certains travaillant finalement au service de leur autopromotion. Là où il avait tort, c'est que nous avions posé d'emblée l'importance des collaborations et du " collectif» (bien avant que ce mot ne revienne dans l'usage) ; la formation de l'Armée noire de Pennequin, bien sûr, mais aussi le boulot qu'on a pu faire, Stéphane Bérard et moi, avec Xavier Boussiron - et bien d'autres.

Et voilà, quelques années plus tard, j'ai eu le sentiment qu'il fallait prononcer les sales mots. Entre autres, pour Tomates (POL, 2010), le nom de Sarkozy : ça a été un déclencheur, un moment de bascule, dans lequel on est encore. Tomates se tisse différemment de ce quej'écrivais auparavant : il y a une volonté d'articulation, de 
transmission, qui coupe avec les poèmes sériels de Grand Ensemble (POL, 2008), avec cette pratique de juxtaposition de blocs de textes que j'avais eue pendant plus de dix ans.

Dans Les Années 10, il y a un très beau texte qui se demande pourquoi les gens d'extrême gauche ne lisent plus de littérature, et vous évoquez la "poésie à effets sociaux", souvent dénigrée, opposée à la poésie qui crée des effets de réel. Comment articulez-vous votre travail de poète par rapport à la politique ?

Je vais partir de deux choses. D'une part, quand je vais sur les sites militants que je fréquente (Paris-luttes.info, et lundimatin par exemple), il y a une nonrelative homogénéité de la prose-même si c'est en train de changer. Heureusement, ça ne ressemble pas du tout à la prose militante des maos... Mais il y a quand même une forme d'homogénéité. Sur un an, il m'est arrivé deux fois seulement de lire des textes qui sonnent autrement: un compte rendu de manifestation, sur Paris-luttes.info, qui n'avait rien de spécialement spectaculaire ou singulier, mais où l'auteur se permettait des échappées de vocabulaire, des points d'exclamation, une expressivité forte mais pas stupide. Tout d'un coup, ça ouvrait les fenêtres, on sentait l'air qui passait. Et je me disais, par contraste: avec les autres textes, on est toujours un peu dans la même pâtée ; ils donnent des informations très précieuses, mais restent rivés à un même ton.

La deuxième chose vient d'une communication que je viens d'entendre sur Hugo Ball, un dadaïste qui était au Cabaret Voltaire en 1916 et qui posait la question suivante: est-ce qu'il peut y avoir une révolution sans formes? (Je ne parle pas des formes institutionnelles, qui s'écriront toujours dans un certain registre.) Mais il est clair que les possibles prennent mieux quand il y a formes, et formes diverses. Je pense à des tracts qui circulent aujourd'hui et éveillent une émotion politique semblable à celle qu'éveille la lecture du tract qu'avaient rédigé Georg Büchner et Friedrich Ludwig Weidig en 1834, Le Messager de Hesse: une émotion devant l'inventivité formelle, l'intelligence, la fermeté du propos. Il y a eu une grande inventivité des tags au moment des manifestations contre la loi travail, mais dans les textes aussi, il faut balancer. Il faut que des poétiques adviennent - ou plutôt, puisqu'elles sont là : il faut éviter les timidités malvenues.

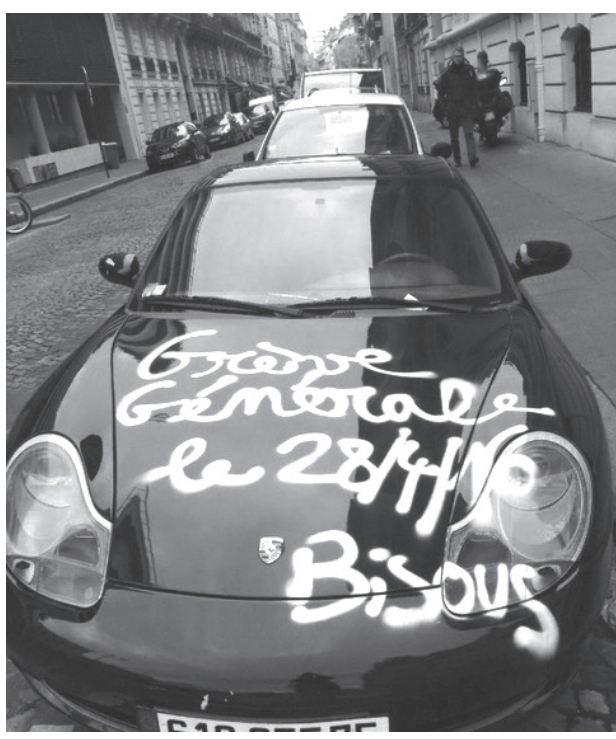

«Grève générale le 28/4/16. Bisous »

Photo d'un des graffitis du mouvement contre la loi travail

Cliché publié sur lundimatin

(<https://lundi.am/Vandalisme-epigraphie>)

Donc, vous avez reproché à une certaine poésie de trop se concentrer sur des questions de formes, mais vous reprochez au discours politique de ne pas assez s'en préoccuper. 
Si je me souviens bien, quand je débute, au début des années 1990, très peu se préoccupent de questions de formes, et encore moins de questions théoriques. Fabriquer de la poésie sans fabriquer en simultané du critique ou une critique, c'est quelque chose quej'ai du mal à concevoir - en héritière déclarée de Rimbaud et de Nerval. L'idée est de garder les deux : garder ce qui s'est mis en place dans les années 1990 et qui provenait d'une rupture avec le discours militant de la génération d'avant: soit un retour à une forme de littéralité, de travail de la langue non dénué d'humour ; mais aussi renouer avec le souci politique. Ce souci est thématisé dans une petite anthologie parue chez Al Dante en 1999, Ouvriers vivants, qui rassemblait des textes de Philippe Beck, Katalin Molnar, Christophe Tarkos... Le réinvestissement nécessaire des questions politiques donne forme cependant qu'une forme travaillée en un sens rend sensible et critique, sensiblement critique. Je crois de moins en moins à un ordre de succession. Je vais vous donner un exemple concret. Je viens de commencer à lire la Première Sécession de la plèbe, livre écrit en 1829 : il raconte le moment où, au ve siècle avant notre ère, la plèbe est montée sur l'Aventin, à Rome, parce que ces hommes du peuple n'en pouvaient plus d'être considérés comme des esclaves. Jacques Rancière a fait une préface à cette réédition, où il explique à peu près ceci : les marxistes considèrent que la lutte mène à l'émancipation, moi je pense que c'est l'émancipation qui mène à la lutte. Mais non! ça se fait concomitamment! Ce n'est pas en renversant une proposition qu'on obtient la vérité ! C'est une facilité de pensée qui peut être très séduisante, et Dieu sait si on l'a utilisée, mais la plupart du temps, les choses se produisent ensemble! Tu mènes une lutte, ça t'émancipe, et donc tu luttes davantage... La littérature, c'est la même chose ! Je suis frappée par ce qui se passe sous Sarkozy, et j'écris ; écrire me conduit à vouloir "sortir» du livre, dont acte; ce passage à l'acte m'amène à écrire, etc. Écrire/agir : l'un renforce l'autre, chauffe l'autre, permet de prendre du recul par rapport à l'autre, dans la réciprocité... Considérer le poème comme une action ne dispense pas d'agir hors poème.

Dans Tomates, le changement de forme passait par le fait d'aller chercher des choses du côté de l'essai, d'hybrider poésie et sciences sociales, mais peut-être aussi par la volonté d'aller chercher un autre lectorat, de s'adresser à plus de monde, contrairement à Grand Ensemble, par exemple, qui est un livre presque crypté...

C'est un texte crypté, en effet, parce que ce qu'il dit ne peut pas encore se dire en France. Je ne peux pas faire autrement, je ne peux pas en parler comme je parle des classes moyennes, et le fait que je ne puisse pas en parler autrement dit quelque chose d'une situation.

Pour le reste, $c^{\prime}$ est une vieille histoire. J'ai eu une passion, très tôt, dès la classe de seconde au lycée, pour les fragments, le romantisme allemand - Novalis, etc. -, mais aussi Lautréamont, Ducasse, Une saison en enfer de Rimbaud : une passion pour ceux qui mélangeaient tout - essai, autobiographie, poésie... J'ai toujours eu ces textes en tête, ce sont eux qui ont déclenché quelque chose. J'ai toujours en tête d'arriver à une sorte de texte déclenchant qui soit de cet ordre-là : où la question poétique n'est pas séparée de la question autobiographique, qui n'est pas séparée de la question politique. C'est le cas chez un poète communard comme 
Rimbaud, ou chez Ernest Courderoy, un romantique frénétique anarchiste qui écrit dans les années 1840. 1848 coupe court à l'élan romantique, la bourgeoisie lâche définitivement les ouvriers; 1848 fait passer, en littérature, à Flaubert: douche froide. Chez Lautréamont, qui s'est fortement inspiré du romantisme frénétique, il y a une forme de surexcitation froide, chauffée à blanc, maintenue, des Chants de Maldoror aux Poésies, qui ne s'opposent pas, ou faussement, parce $q^{\prime}{ }^{\prime} a u$ fond c'est le même ton.

\section{C'est quoi, le "peuple", pour vous?}

Je ne sais toujours pas s'il vaut mieux dire ou écrire "plèbe» plutôt que « peuple » ou autre chose. Je ne sais même pas si «peuple» ne se reconnaît que dans l'événement, et se dissout corps et biens lorsque celui-ci est passé. Blanchot, en 1968, écrit: "Nous sommes tous la pègre! » - il choisit "pègre » plutôt que " peuple » ou même "plèbe ", sans doute en partie parce qu'il s'agit pour lui de descendre encore plus bas, d'être plus «radical» que tous les autres; ou que pour lui «pègre» est le nom d'un peuple désœuvré... Le substantif a son importance, mais le travail que j'ai essayé de mener dans Tomates et dans le texte sur les pauvres (dans Les Années 10) s'attache plutôt aux pronoms et aux prépositions, dans un jeu où la seule solution provisoire est de glisser de la première personne $\mathrm{du}$ singulier à la première du pluriel via la troisième... sans arrêt nulle part, puisqu'on ne sait jamais s'il vaut mieux parler pour les pauvres, avec ou par ou sans, ou s'il vaudrait mieux se taire.

Revenons au rapport entre l'écriture et l'action. Comment penser l'efficace littéraire?
L'efficace littéraire ne peut pas être pensée seule, ça ne donne rien : le problème, c'est ce qui se passe autour. Est-ce que la situation historique est chaude, suffisamment, pour que la littérature prenne sens là-dedans? On peut poser la question de l'efficace de l'art si le reste se met à être un peu plus efficace aussi, et déverrouillé. Je ne crois pas vraiment à une avant-garde: les dadaïstes, les surréalistes, ils étaient là parce que ça chauffait partout à l'époque. Il y avait autour des révolutions qui se faisaient ou s'étaient faites, et le principal sujet de discussion, entre Artaud et Breton par exemple, portait sur l'ampleur et la qualification de la révolution, non sur sa nécessité, qui était actée : adhésion au PC d'un côté, soit révolution prolétarienne avant toutes choses ; désir d'une "révolution intégrale » de l'autre - qui n'excluait ni le matérialisme ni la révolution prolétarienne, d'ailleurs, mais se souciait d'un changement de l'existant dans son entier. En 1936, quand il est au Mexique, Artaud explique très clairement ce qu'il entend, lui, par «changer la vie», dans une perspective qu'on dirait aujourd'hui "décoloniale», anti-patriarcale et antiautoritaire. Sur ces trois points, ses conférences de l'époque et ses "Messages révolutionnaires » sont explicites. Il y a des moments où les poètes peuvent être explicites, ce qui ne signifie pas, en tout cas certainement pas pour Artaud, lâcher une densité syntaxique. Artaud, c'est pas compliqué : c'est le plus intelligent. On le dit trop peu, je trouve.

Il me semble que l'efficace littéraire passe, entre autres, par une hygiène de la reprise - par qui telle formule, telle phrase, est reprise, pour qui, à destination de qui, et à quel moment. Soit «changer la vie», justement. La phrase 
apparaît dans une lettre de Rimbaud, elle relève de la correspondance privée ; elle n'est pas là pour paraître en Pléiade cent ans plus tard. Mais les textes sont redécouverts, les formules rimbaldiennes sont extraites, reprises par Dada, par le surréalisme, et en 1968. C'est là qu'on peut parler d'efficace, d'une efficacité immédiate, visible, $d$ 'une rencontre entre un bonheur d'expression et la joie de l'insurrection, en quelque sorte. Certes, la même formule peut être reprise par Auchan, transformée en slogan publicitaire, illustrée par une image de semoule. Simplement ce n'est pas le problème: Macron écrit bien un livre qui s'appelle Révolution! Et alors? On ne va pas rentrer chez soi et fermer les volets pour autant. Bien sûr, il y a de la récupération. Mais le problème reste : et nous, qu'est-ce qu'on en fait? Comment, nous, nous le reprenons, et comment la force et l'inventivité qui nous précèdent et se conservent dans les textes et dans les mémoires nous relancent, et si oui ou non nous sommes encore capables de nous en ressaisir pour être à notre tour inventifs et forts face à l'adversité. Je crois que les tags et les banderoles du printemps 2016 apportent une réponse : oui, on en est parfois plutôt capables. On sait faire.

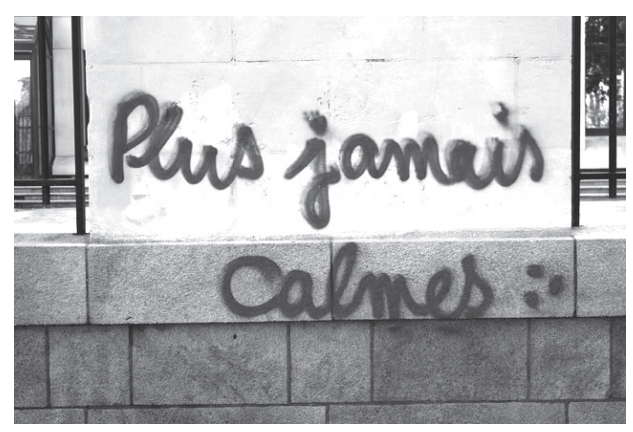

«Plus jamais calmes ». Nantes, novembre 2017 Cliché de Suvann publié sur La rue ou rien (<http://larueourien.tumblr.com/>)
Dans votre refus de l'avant-garde, il y a la conviction qu'être moderne, c'est être au présent, qu'il y a une circulation nécessaire entre l'action politique et les possibilités de la littérature.

Dans Rimbaud, la Commune de Paris et l'invention de l'histoire spatiale (Les Prairies ordinaires, 2013), Kristin Ross relit Rimbaud par la Commune. De même dans Juin 1848, le spleen contre l'oubli (La Fabrique, 2017), Dolf Oehler relit Flaubert, Baudelaire, en partant de la rupture que constitue juin 1848. Il montre comment relire certains poèmes de Baudelaire en pensant aux massacres de juin, et aux effets de censure qui ont suivi : on peut dire que la modernité naît là aussi, dans le corset impérial, et dans un désespoir sans pitié aucune, dans une ruse maligne aussi : avec Baudelaire, on sent que s'ouvre quelque chose qui n'est plus négociable ; il y aura bien quelques gentils poètes encore, mais ceux-là, on les a tous oubliés ; ceux dont on se souvient, ce sont les méchants! Baudelaire, Rimbaud, Artaud plus tard; y a plus de place pour la parnasserie, ni pour «le décharné bien écrit» (ça, c'est d'Artaud!), ni pour les jeux poétiques sympa; la poésie n'est pas là pour consoler ou "prendre soin", comme on dit aujourd'hui - en tout cas, certainement pas celle de Baudelaire. Pour Dada, c'est pareil ! La première fois que Hugo Ball monte sur scène pour dire un poème phonétique, c'est-à-dire un poème sonore, des vers sans mots, c'est parce qu'ils se sont tapé quatre ans de propagande, de bouillasse intellectuelle totale, que raconte très bien Proust dans Le Temps retrouvé, et qu'ils n'en peuvent plus. Il faut que ça pète de ce côté-là aussi! Les premières performances dada ont lieu en pleine guerre, en 1916 : 
ils n'en peuvent plus, ils disent: "ça suffit vos discours qui nous ont conduits à la catastrophe, bande de dégénérés ». Ce n'est pas une crise d'adolescence !

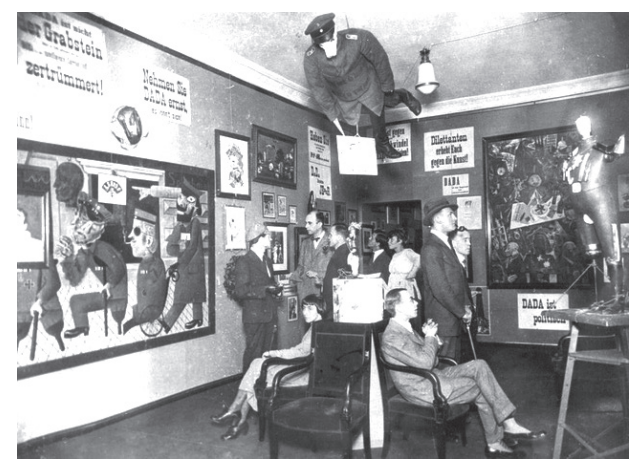

Première foire internationale Dada. Berlin, 1920

La question de l'efficacité poétique a pu se penser aussi dans le cadre de la performance, que vous avez beaucoup pratiquée par le passé.

J'ai fait beaucoup de performances entre 1994 et 2004, jusqu'à Tomates : tu arrives, tu balances le truc - personne ne disait bonjour -, et puis ça s'arrête. Pendant dix ans, on a tous fait ça, c'était le format "Polyphonix» (festival créé par Jean-Jacques Lebel), le format qu'on imaginait être celui des avant-gardes historiques, leur héritage. En fait, ça annonçait, je crois, la manière dont les institutions de l'art (les écoles, les musées, les fondations...) se sont ressaisies de la performance : une forme artistique close, une "pièce" à enregistrer et archiver, un répertoire, un objet d'étude, une histoire monnayable (qu'on pense à Marina Abramovic). Le tournant, c'est le début des années 2000 : maintenant, il suffit que vous leviez le petit doigt dans une galerie pour que ce soit podcastable ad vitam! C'est pour ça qu'il y a aujourd'hui de gros enjeux autour de la performance et de la lecture publique, justement parce qu'elles sont coincées entre leur capture par certaines institutions de l'art contemporain, et donc l'éternel soupçon que tout ça, c'est juste du spectacle et de l'autopromotion, et un geste directement politique dans la mesure où le public est là, où l'adresse au public est lisible/visible/audible en tant qu'adresse, et où sont soulevés là (ici et maintenant) les problèmes de ce public, des problèmes auxquels est confronté le poète, bien sûr, des problèmes d'usages et d'habitudes.

Mais à l'époque, en 2004, j'avais pas vu tout ça: j'ai arrêté parce que j'étais fatiguée de faire de longs trajets pour un quart d'heure de perf, et puis on était toujours les mêmes à intervenir, ça tournait à cinq ou six : les jeunes poètes aujourd'hui sont plus nombreux. Or on fait ça aussi pour rencontrer des gens, pour discuter! Depuis Tomates, la place de ceux qui viennent écouter est de plus en plus importante. Si ça ne se termine pas en discussion, je suis un peu déçue : c'est un moyen de mettre une boussole, de nous indiquer, ensemble, où est le nord. On a impérativement besoin de se parler, il ne faut pas craindre de palabrer. Il ne faut pas avoir peur que ce ne soit pas efficace. Les gens qui redoutaient que Nuit debout ne soit pas « efficace", ils sont cons. Je comprends que certains aient voulu tout de suite s'organiser, faire une constitution, mais en même temps il fallait commencer par se parler : ça fait quarante ans qu'on ne se parle pas, il y a un temps de réadaptation! Aujourd'hui, si je fais des interventions qui sont entre la lecture et la conférence, c'est pour essayer d'amener à la discussion.

Parler de l'articulation entre écriture et politique, c'est aussi parler de politique 
éditoriale : pourquoi avoir publié Les Années 10 à La Fabrique?

C'était une commande, au départ. J'aurais très bien pu intervertir les publications entre POL et La Fabrique. Par exemple, cette année, je sors un essai sur Proust à La Fabrique et une sorte de chronique politique chez POL; "logiquement », ça aurait dû être l'inverse. Il ne s'agit pas de dérouter le lectorat !... mais d'insister encore sur l'idée qu'une certaine littérature, ou poésie, peut être un acte politique, que réflexion sensible et excitation politique sont liées, et se renforcent l'une l'autre d'être liées. Il faut répéter ça.

\section{Note}

1 Sur cette posture, de Hugo à Tel Quel, voir ici même la lecture que fait Marik Froidefond
En tout cas, publier à La Fabrique m'a visiblement donné une forme de «légitimité » aux yeux de certains, cela m'a permis de rencontrer des gens que je n'aurais jamais rencontrés avant. C'est quand même terrible. Tomates, qui était paru chez POL, était presque un appel du pied adressé aux gens qui discutent de politique. Mais il fallait le changement d'éditeur pour que ces gens-là s'autorisent à lire ce que j'écris, à lire cette littérature-là ; parfois à lire ou relire de la littérature tout court. $\mathrm{Y}$ a du boulot! On est très engoncés, en France, très coincés... Vieille époque, vieux réflexes, le tout peinturluré en neuf... mais qui ça trompe?

du livre de Laurent Jenny, Je suis la révolution (p. 197-200). 